\title{
Article \\ Effect of Conventional and Electronic Cigarettes Smoking on the Color Stability and Translucency of Tooth Colored Restorative Materials: An In Vitro Analysis
}

\author{
Hamad A. Alnasser ${ }^{1}\left({ }^{\circ}\right.$, Ahmed A. Elhejazi ${ }^{2}{ }^{\circ}$, Abdalrahman A. Al-Abdulaziz ${ }^{3}{ }^{\circledR}$, Saad S. Alajlan ${ }^{4}$ \\ and Syed Rashid Habib ${ }^{5, *}$ (D) \\ 1 Ministry of Health, Riyadh 13536, Saudi Arabia; hamad.alnasser94@gmail.com \\ 2 Department of Restorative Dental Science, King Saud University, Riyadh 11545, Saudi Arabia; \\ aelhejazi@ksu.edu.sa \\ 3 Medgulf Insurance Company, Riyadh 12444, Saudi Arabia; abdalrahman.992a@gmail.com \\ 4 Dr. Sulaiman Alhabib Hospital, Riyadh 11445, Saudi Arabia; saadalajlan0@gmail.com \\ 5 Department of Prosthetic Dental Sciences, College of Dentistry, King Saud University, \\ Riyadh 11545, Saudi Arabia \\ * Correspondence: syhabib@ksu.edu.sa; Tel.: +966-53-475-0834
}

check for updates

\section{Citation: Alnasser, H.A.;}

Elhejazi, A.A.; Al-Abdulaziz, A.A.; Alajlan, S.S.; Habib, S.R. Effect of Conventional and Electronic

Cigarettes Smoking on the Color Stability and Translucency of Tooth Colored Restorative Materials: An In Vitro Analysis. Coatings 2021, 11, 1568. https://doi.org/10.3390/ coatings11121568

Academic Editors: João Paulo Mandes Tribst and Roberto Lo Giudice

Received: 19 November 2021 Accepted: 14 December 2021 Published: 20 December 2021

Publisher's Note: MDPI stays neutral with regard to jurisdictional claims in published maps and institutional affiliations.

Copyright: (c) 2021 by the authors. Licensee MDPI, Basel, Switzerland. This article is an open access article distributed under the terms and conditions of the Creative Commons Attribution (CC BY) license (https:// creativecommons.org/licenses/by/ $4.0 /)$.

\begin{abstract}
This in vitro study compared the effects of conventional and electronic cigarettes on the aesthetics (color stability and translucency) of two types of composite resins: micro and nano-hybrid. Methods: A total of 120 specimens from two different composite materials Filtek Z250 XT (Nanohybrid, 3M) and Filtek Z250 (Micro-hybrid, 3M) were divided into four groups ( $\mathrm{n}=30$ ); shade A2 was used. The samples were exposed to conventional and electronic cigarette smoke via a custom made chamber device. The color values and measurements were recorded using a spectrophotometer before and after the exposure. The color and translucency were evaluated using the three-dimensional CIE Lab. Results: There was a significant change in the color $(\Delta \mathrm{E})$ and the translucency parameter (TP) in all of the specimens exposed to electronic cigarettes and conventional cigarettes. The results showed that the highest $\Delta \mathrm{E}$ mean is for the nano-hybrid composite exposed to conventional cigarettes with $1.74 \Delta \mathrm{E}$ while the same material is 0.64 under the electronic cigarettes and the difference is significant with $(p<0.05)$. The micro-hybrid composite data showed less changes in color under both exposures with $0.85 \Delta \mathrm{E}$ mean under the conventional cigarette smoke and 0.48 under the electronic cigarette smoke with $(p<0.004)$. Conclusions: The conventional cigarette smoke has more effect on the color stability of the composite resins than electronic cigarettes. From a clinical point of view, the effect of smoke exposure on the tested specimens' color, for the time duration to which the specimens were exposed, were moderate $(\Delta \mathrm{E}<2)$. The micro-hybrid composites showed better color stability as compared to the nano-hybrid composites.
\end{abstract}

Keywords: color; composite; smoking; conventional cigarette; resin; electronic cigarette

\section{Introduction}

The aesthetic demand from patients has increased in the past few decades. Composite resins are the preferred materials for direct restorations [1]; however, regardless of the outstanding improvement and development of the aesthetic and mechanical properties of resins, some deficiencies still exist [1-3]. Restorations that are exposed to some contents of the conventional cigarettes, such as carbon monoxide and ammonia, become yellow or even black and there is a great change in their color [4]. Nowadays, tooth-colored restorative materials have the ability to mimic the natural appearance of the tooth enamel and dentin, but unfortunately even the best restorations available are prone to staining and their optical behavior and color stability can be affected [5]. Many factors could cause extrinsic staining to the tooth-colored restorations such as tea, coffee, acidic beverages, and the nicotine in cigarettes [6]. Cigarette smoking changes the color and surface texture of 
composite leading to increased staining. Polishing decreases superficial staining, but it may not return the composite to its original color [7].

The most common color parameters that help in choosing the dental ceramics from color/shade perspective and for the evaluation/comparison of color differences are the Delta $\mathrm{E}(\Delta \mathrm{E})$ and Translucency $[8,9] . \Delta \mathrm{E}$, which is also termed as the total-color-difference, is obtained from calculation based on $\mathrm{L}^{*}, \mathrm{a}^{*}, \mathrm{~b}^{*}$ values. The advantage of $\Delta \mathrm{E}$ is that it quantifies the combined color/shade differences between the various esthetic materials with a numerical number, and makes the comparisons be-tween these materials simple, convenient, and easy [9-11]. Further to $\Delta \mathrm{E}$, the dental ceramics translucency is also considered as important clinical parameter for classifying the esthetic materials into esthetic/non-esthetic and is obtained by calculating the difference between the $L^{*} a^{*} b^{*}$ values of the test materials over white/black background at a standardized thickness, termed as TP [12,13].

Electronic cigarettes (E-Cigarettes) known as vapes were first introduced to the market in the early 2000s and their use has been increasing since then due to the claims of their safety and their ability to help in smoking cessation [14-16]. Almost 750,000 E-Cigarettes were sold in 2010 reaching 3.5 million in 2012 and there is a strong expectation that the numbers of sales will continue to increase globally [17]. E-Cigarettes are battery-powered gadgets that try to mimic the act of conventional smoking and it allows the users to inhale nicotine aerosol without other cigarette content such as carbon monoxide, tar, ammonia, nickel, and cadmium [8]. One study showed an inquiry with the Food and Drug Administration (FDA) for nicotine amount and impurities quantitation in two ECigarette brands and results demonstrated low nicotine content [18]. Some other studies reported in aerosols presence of aldehydes and acroleins as a result of VG and PG heating, metals presence in fluids, and aerosol from "cartomizer-combination of atomizer and cartridge in new E-Cigarette model" fibrous pads and volatile organic compounds (VOCs) identification including xylene, benzene, styrene, and toluene presence after chemical analysis in both aerosol and liquids [10-12].

The effect of conventional smoking on the color change of composite resins has been reported in several studies [19-22]. Additionally, conventional smoking affects the translucency of dental enamel and resin composites [23,24]. Translucency is the ability of a layer of a colored substance to allow an underlying background to show through. RBCs are optically translucent materials because of their structure, which is composed of a highly transparent matrix and small filler particles [25]. Translucency affects the depth of color in the restorations and influences the aesthetic harmonization with surrounding or adjacent teeth and restorations [23]. Since the use of E-Cigarettes substantially increased in past few years, currently, there is a lack of research on the effects of E-Cigarettes on the color stability and translucency of tooth-colored restorative materials. Therefore, the aim of this in vitro research study was to compare the effects of conventional and electronic cigarettes on the aesthetics (color stability and translucency) of two types of composite resins, i.e., micro and nano-hybrid. The null hypothesis was that there is no significant difference in the color and translucency of the two investigated composite materials after exposure to smoke of conventional and electronic cigarettes.

\section{Materials and Methods}

\subsection{Study Design and Sample Size Calculation}

In this in vitro study, a total of 120 specimens from two different commercially available composite materials were selected. Using sample size calculator software (SPSS, 2022, IBM, Chicago, IL, USA) with $\alpha$ (level of significance) set at 0.5 , effect size of 0.35 and power of 0.9, the total sample size calculated was 120. A total of 60 Nano-hybrid Filtek Z250 XT (3M ESPE, Saint Paul, MN, USA) and 60 Micro-hybrid Filtek Z250 (3M ESPE, Saint Paul, MN, USA), (shade A2) were used (Table 1).

The samples were divided into four groups $(\mathrm{n}=30)$ and randomly assigned according to the type of smoke that they were exposed to:

Group 1-Z250 XT composite that was exposed to conventional cigarettes. 
Group 2-Filtek Z250 composite that was exposed to conventional cigarettes.

Group 3-Z250 XT composite that was exposed to electronic cigarettes' smoke.

Group 4-Filtek Z250 composite that was exposed to electronic cigarettes.

Table 1. Composition of the restorative materials used in the study.

\begin{tabular}{cc}
\hline Materials (Shade A2) & Composition \\
\hline Nano-hybrid composite filtek (Z250 XT). & $\begin{array}{c}\text { Bis-GMA, UDMA, TEGDMA, BIS-EMA, 55-57\% of } \\
\text { filler: Barium glass, ytterbium trifluoride, mixed } \\
\text { oxides, and silica dioxide particles. }\end{array}$ \\
\hline Micro-Hybrid composite feltick (Z250). & $\begin{array}{r}\text { Bis-GMA, TEGDMA, UDMA, BisEMA, 60\% of filler: } \\
\text { zirconium/silica particles. }\end{array}$ \\
\hline
\end{tabular}

\subsection{Specimen Preparation}

A stainless-steel mold (10-mm in diameter and 2-mm in thickness) was used to prepare the specimens and a matrix strip (Mylar, Dupont, Wilmington, DE, USA) was placed over the mold and light pressure was applied on the matrix to remove any excess composite and to make the outer layer of the composite as smooth as possible then the specimens were placed on a glass slide after that they were light-cured with (3M Elipar ${ }^{\mathrm{TM}}$ Deep Cure-S LED, Saint Paul, MN, USA) for $40 \mathrm{~s}$ for each specimen at $2.5 \mathrm{~cm}$ distance from the specimens and power output of $1.470 \mathrm{~mW} / \mathrm{cm}^{2}$ (Figure 1). The specimens were stored in distilled water at room temperature $24 \mathrm{~h}$ before the exposure to cigarettes.

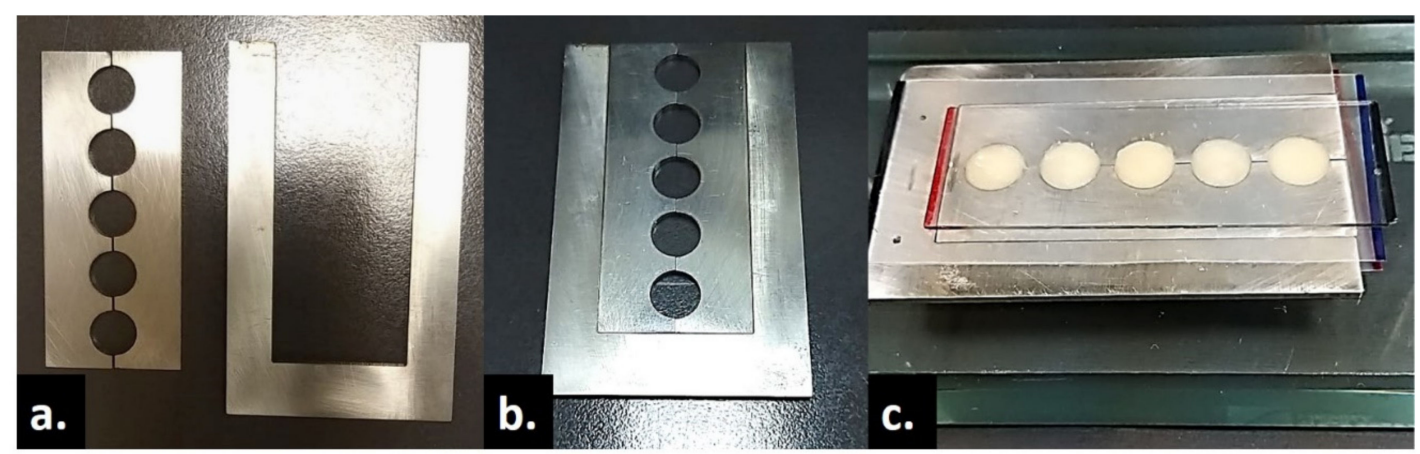

Figure 1. (a,b) Stainless-steel mold used for preparation of specimens; (c) composite specimens being prepared using the stainless-steel mold.

\subsection{Exposure of Specimens to Smoking}

Custom made chamber device was made consisting of a vacuum system that directed the smoke to a custom-made plastic box (Figures 2 and 3). The specimens were stabilized by polyvinyl siloxane putty index (manufacturer) which was placed inside the box at $8 \mathrm{~cm}$ distance from the smoke. The specimens were placed vertically on both sides of the customized chamber in order to mimic the intraoral scenario of restorations getting exposed to the smoking. Horizontal placement of the specimens was avoided intentionally to avoid unrealistic adherence of smoking dust to the specimens.

Conventional cigarettes (Marlboro, Philip Morris International Inc., New York, NY, USA) and electronic cigarette (Greensound LTD, GS Q80, Shenzhen, China) were used in the experiments. The specimens were exposed to cigarette smoke for $90 \mathrm{~min}$ (90 cigarettes). Then the specimens were gently washed with distilled water for $1 \mathrm{~min}$, and the final color was measured with the spectrophotometer after $2 \mathrm{~h}$. The E-Cigarettes batteries were fully charged and stored at room temperature. The vacuum flow rate to pull aerosol out of atomizer was $20 \mathrm{~mL} / \mathrm{min}$. This flow rate allowed aerosol capture in first trap without moving on to second trap and prior to aerosolization, none of the E-Cigarette non-aerosolized e-liquid was pulled out. 


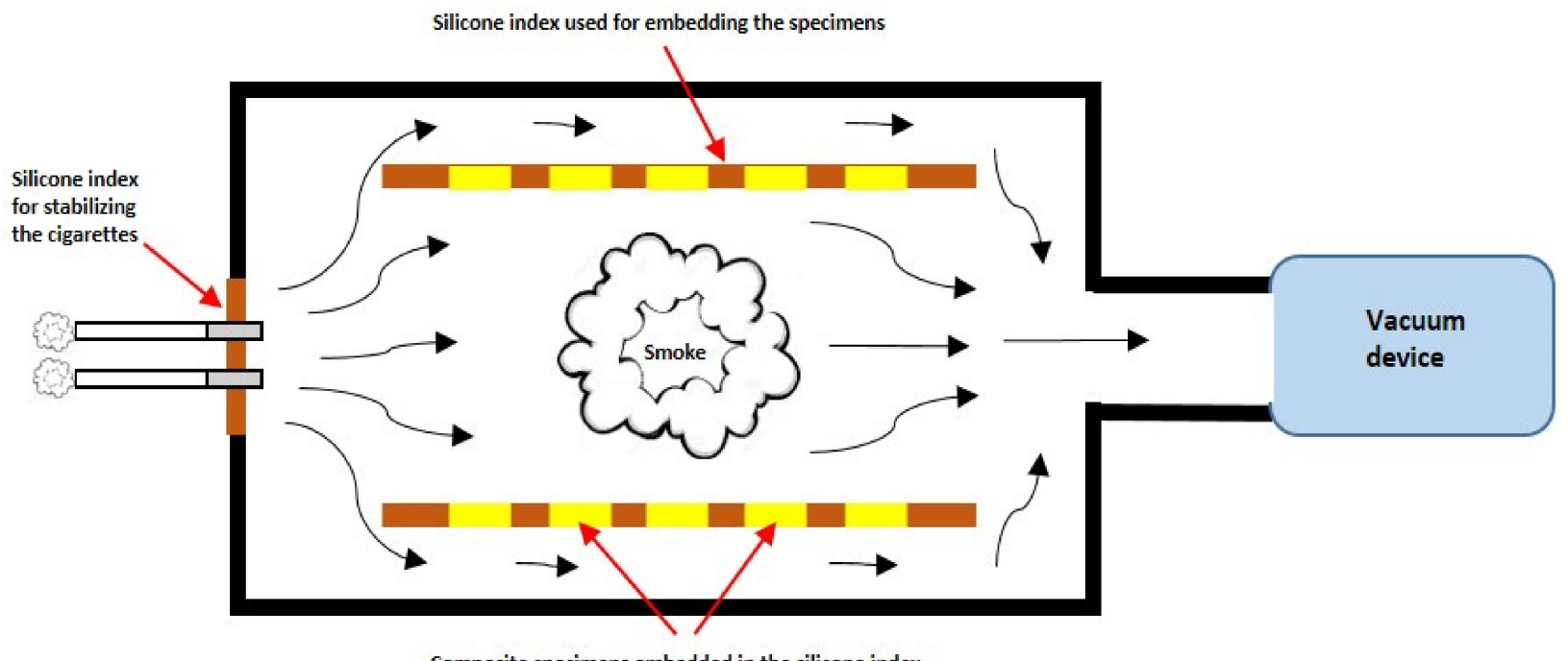

Composite specimens embedded in the silicone index

Figure 2. Schematic representation of the process of the experiment.

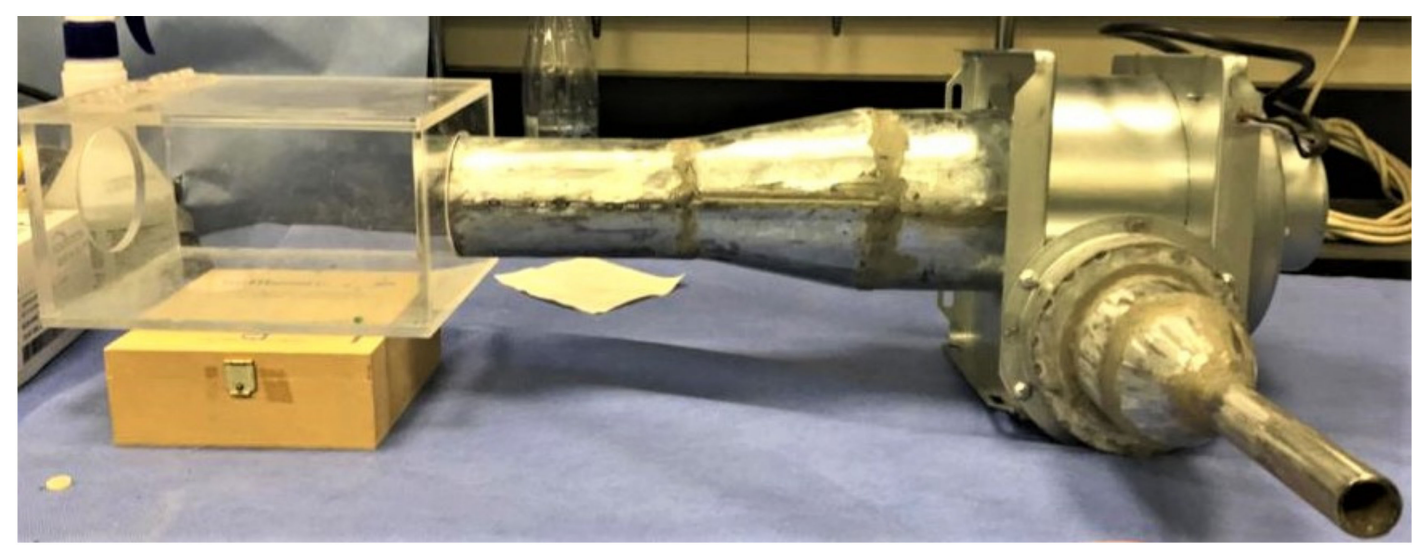

Figure 3. Customized chamber with suction device.

\subsection{Color Measurements}

The color measurements were taken by CIELab color system and $(\Delta \mathrm{E})$ was calculated before and after the experiment via the spectrophotometer (Hunterlab, Labscan XE, Reston, VA, USA) with automated Variable Sample Illumination and target mask opening of $44 \mathrm{~mm}$. There are two chromatic axes, $a^{*}$ (red-green parameter difference) and $b^{*}$ (yellow-blue parameter difference), which are at right angles to one another, representing the saturation level and hue dimensions. The third axis, $\mathrm{L}^{*}$, perpendicular to the chromatic planes represents the value or lightness. The CIELab color difference $(\Delta \mathrm{E})$ was calculated as follows [9]:

$$
\Delta \mathrm{E}=\left[\left(\Delta \mathrm{L}^{*}\right)^{2}+\left(\Delta \mathrm{a}^{*}\right)^{2}+\left(\Delta \mathrm{b}^{*}\right)^{2}\right]^{1 / 2}
$$

To measure the translucency, the color of all the specimens was measured using black and white backgrounds before and after the experiment and the translucency parameter $\mathrm{TP}$ to detect the differences was calculated according to this formula:

$$
\mathrm{TP}=\left[\left(\mathrm{L}^{*} \mathrm{~B}-\mathrm{L}^{*} \mathrm{~W}\right)^{2}+\left(\mathrm{a}^{*} \mathrm{~B}-\mathrm{a}^{*} \mathrm{~W}\right)^{2}+\left(\mathrm{b}^{*} \mathrm{~B}-\mathrm{b}^{*} \mathrm{~W}\right)^{2}\right]^{1 / 2}
$$

where $\mathrm{W}$ is against the white background and $\mathrm{B}$ is against the black background [9]. 


\subsection{Statistical Analysis}

All the data were tabulated and analyzed by Statistical package for social sciences (SPSS; Version 23; SPSS Inc., Chicago, IL, USA). Descriptive stats for mean L*a*b* values, calculation of $\triangle \mathrm{E}$ and TP from the $\mathrm{L}^{*} \mathrm{a}^{*} \mathrm{~b}^{*}$ values were recorded and tabulated for the test composite materials. The data were found to be normally distributed according to Kolmogorov-Smirnov test. The formal analysis was carried out, comparing the mean values of $\Delta \mathrm{E}$ and TP (95\% CIs) of all the five groups using Paired Samples t test at probability of $\alpha<0.05$.

\section{Results}

It was verified that all four groups presented a significant change in color $\Delta \mathrm{E}$ under exposure to both the electronic and conventional cigarettes and they became darker in color. Table 2 presents the results of paired samples $t$ test with the mean $\Delta \mathrm{E}$ for each test group. The results showed that the highest $\Delta \mathrm{E}$ mean is for the nano-hybrid composite exposed to conventional cigarettes with $1.74 \Delta \mathrm{E}$ while the same material is 0.64 under the electronic cigarettes and the difference is significant with $(p<0.05)$. The micro-hybrid composite data show less changes in color under both exposures with $0.85 \Delta \mathrm{E}$ mean under the conventional cigarette smoke and 0.48 under the electronic cigarette smoke with $(p=$ 0.004). These data revealed that the conventional cigarettes have more effect on the color change of composite materials than electronic cigarettes.

Table 2. Comparing smoking within each type of composite.

\begin{tabular}{cccccc}
\hline Composite & Smoking & $\mathbf{N}$ & Mean & Std. Deviation & $p$-Value \\
\hline \multirow{2}{*}{ Nano } & Conventional & 30 & 1.714 & 1.166 & \multirow{2}{*}{0.000} \\
& Electronic & 30 & 0.646 & 0.301 & \multirow{2}{*}{0.004} \\
\hline \multirow{2}{*}{ Micro } & Conventional & 30 & 0.8512 & 0.589 & 0.262 \\
& Electronic & 30 & 0.487 & \\
\hline
\end{tabular}

Table 3 shows that micro-composite group specimens had more color stability than the nano-composite $(p<0.05)$ under conventional smoking and less significant change under electronic smoking with $(p=0.033)$. Furthermore, the results revealed that the electronic cigarettes affected both tested materials to similar levels while conventional cigarettes affected the color and caused more staining for the nano-composites as compared to the micro composites.

Table 3. Comparing the type of composite with each type of smoking.

\begin{tabular}{lccccc}
\hline Smoking & Composite & $\mathbf{N}$ & Mean & Std. Deviation & $p$-Value \\
\hline \multirow{2}{*}{ Conventional } & Nano & 30 & 1.714 & 1.166 & 0.001 \\
& Micro & 30 & 0.646 & 0.301 & \multirow{2}{*}{0.033} \\
\hline \multirow{2}{*}{ Electronic } & Nano & 30 & 0.8512 & 0.589 & 0.262 \\
& Micro & 30 & 0.487 & \\
\hline
\end{tabular}

With respect to the three-dimensional CIE Lab system, the value of $\mathrm{L}^{*}$ axis which represents the lightness decreased in all groups. The nano-composite became greener and more bluish while the micro-composite became more reddish and yellowish (Table 4).

Regarding the translucency, there was a significant change in the values of the translucency parameter before and after the experiment. Table 5 shows the results of the t paired test with a significant level of $(\alpha=0.05)$. The mean of the TP reduced after the experiment in all groups. The first group of the nano-hybrid composite that was exposed to conventional cigarettes reduced from 10.4 TP before the exposure and 7.4 after the exposure with $(p=0.006)$. Additionally, the second group of micro composites exposed to conventional smoking reduced from 9.9 TP before the exposure and 6.9 after it with $(p=0.031)$, so the nano-hybrid were more affected than the micro-hybrid. The groups exposed to electronic 
cigarettes also experienced a significant decrease in the TP values but it was less than the gropes exposed to conventional cigarettes, thus the null hypothesis is rejected and we observed significant change in translucency and color after exposure to E-Cigarettes in restorative materials used.

Table 4. CIE LAB System values.

\begin{tabular}{lccccccc}
\hline \multicolumn{8}{c}{ Mean of $\mathbf{L}$, a and $\mathbf{b}$ Axis before and after } \\
\hline \multirow{2}{*}{ Smoking } & Composite & $\mathbf{L}^{*}$ before & $\mathbf{a}^{*}$ before & $\mathbf{b}^{*}$ before & $\mathbf{L}^{*}$ after & $\mathbf{a}^{*}$ after & $\mathbf{B}^{*}$ after \\
\hline \multirow{2}{*}{ Conventional } & Nano & 58.67 & -1.66 & 5.03 & 57.88 & -2.23 & 4.75 \\
& Micro & 54.72 & -2.45 & 3.543 & 54.11 & -2.25 & 3.79 \\
\hline \multirow{2}{*}{ Electronic } & Nano & 57.4 & -1.83 & 4.16 & 57.07 & -2.013 & 4.01 \\
& Micro & 54.53 & -2.44933 & 3.371 & 54.49 & -2.268 & 3.53 \\
\hline
\end{tabular}

Table 5. The effect of the smoke on translucency.

\begin{tabular}{|c|c|c|c|c|c|}
\hline Smoking & Material & Translucency Parameter (TP) & $\mathbf{N}$ & Mean & $p$-Value \\
\hline \multirow{4}{*}{$\begin{array}{l}\text { Conventional } \\
\text { smoking }\end{array}$} & Nano-hybrid & Before & 30 & 10.408 & \multirow{2}{*}{0.006} \\
\hline & Composite & After & 30 & 7.468 & \\
\hline & Micro-hybrid & Before & 30 & 9.921 & \multirow{2}{*}{0.031} \\
\hline & Composite & After & 30 & 6.907 & \\
\hline \multirow{4}{*}{$\begin{array}{l}\text { Electronic } \\
\text { smoking }\end{array}$} & Nano-hybrid & Before & 30 & 10.518 & \multirow{2}{*}{0.129} \\
\hline & Composite & After & 30 & 7.418 & \\
\hline & Micro-hybrid & Before & 30 & 9.791 & \multirow{2}{*}{0.174} \\
\hline & Composite & After & 30 & 6.528 & \\
\hline
\end{tabular}

\section{Discussion}

The present study evaluated and compared the effects of conventional and electronic cigarettes on the color stability and translucency of micro and nano-hybrid composites. A unique method of exposing the specimens to the smoke of cigarettes in a custom designed smoking chamber was employed in the study. The results of the study revealed significant change in translucency and color of the tested restorative materials after exposure to the cigarettes, thus rejecting the null hypothesis.

In the present study specimens were fabricated from two resin composites Nanohybrid Filtek Z250 XT (3M ESPE, Saint Paul, MN, USA) and Micro-hybrid Filtek Z250 (3M ESPE, Saint Paul, MN, USA) as per manufacturer's instructions to examine the effect of conventional and electronic cigarettes use on their color stability and translucency. Both composites were selected due to developed reputation in restoration stabilization in oral environment due to their high quality [26]. Nano-hybrid Filtek Z250 XT restorative composites are found beneficial in attaining longevity of restorations as they show similar "mean flexural modulus (17.44 GPa)" to "dentin flexural modulus (19 GPa)" [27-29] the other used restorative resin composite is Micro-hybrid Filtek Z250 (3M ESPE, Saint Paul, MN, USA) claimed to decrease polymerization shrinkage and improve resin efficacy in class I restorations due to use of monomers i.e., mixture of TEGDMA and Bis-GMA in addition to Bis-EMA or UDMA.

Nowadays the younger generation/youth are very careful and possessive about the appearance and the whiteness of their teeth. There is also an increase in the rate of consumption and use of the products that cause extrinsic staining such as coffee, acidic beverages, and smoking [10]. Smoking is responsible for so many health consequences and most of them do not show until late adulthood [30]. Smoking affects the oral health too. A study found out that smoking is associated with the increase of both severity and the prevalence of the periodontal disease; another study in the United States found out that $41 \%$ of people with periodontitis are current smokers and $10 \%$ are previous smokers [14,31]. Electronic cigarettes were found to be helping people in smoking cessation and people 
who smoked them for more than 2 years had a higher chance in smoking cessation than those who used them for a short period of time. Additionally, the electronic cigarettes help in reducing the number of conventional cigarette smoked per day [14-16]. However, the current data showed that the use of electronic cigarettes posed a different type of effect on the color of restorations compared to conventional cigarettes. Some people use both electronic cigarettes and conventional cigarettes at the same time and this increases the rates of morbidity and mortality [13].

Ideally the differences in the composition of the aesthetic restorative composites available in the market should behave similarly to any extrinsic staining and exhibit no difference in their color to these stains [32]. The results of this study revealed significant changes in the color of the investigated composite materials with regards to the changes observed in the $\Delta \mathrm{E}$ and the translucency values. The total color difference or $\Delta \mathrm{E}$ is considered as a standardized tool for evaluation and comparison of color changes in color science. According to the color changes in aesthetic restorations the $\Delta \mathrm{E}<1$ cannot be detected by the naked human eye and if $1<\Delta \mathrm{E}<3.3$ can be detected only by an experienced dentist and finally $\Delta \mathrm{E}>3.3$ can be detected easily by anyone and it is unacceptable clinically $[9,10]$. While it is true that all values of $\Delta \mathrm{E}$ in this study increased it is also true that all of them were $<3.3$ which makes them clinically acceptable. One of the reasons for this was the small distance of $2.5 \mathrm{~cm}$ used. On the other hand, in a previous study done on Nano-hybrid composite Filtek (Z250 XT) exposed to conventional cigarettes, the mean value after the exposure was $24 \Delta \mathrm{E}$ which is much higher than this study's value of $1.7 \Delta \mathrm{E}$. They claimed that their high values are due to using cigarettes with dark components, and the distance that was between the samples and the exposure was $1 \mathrm{~cm}$, which might play a big role in that high value, if we compare it with this study's distance $(8 \mathrm{~cm})$ [4]. Another study done on a bovine enamel exposed to electronic cigarettes showed a mean value of $3.3 \Delta \mathrm{E}$ and it is more than this study's values $0.64 \Delta \mathrm{E}$ for the nano-hybrid [1]. The study used an exposure time of $70 \mathrm{~min}$ which is $20 \mathrm{~min}$ less than the time used in this study, so it can be observed that if we increase the exposure time it will produce more similar results [1]. A study exposed the nano-hybrid composite Filtek (Z250 XT) to conventional smoke and the mean value was $9.7 \Delta \mathrm{E}$. This study also used a $1 \mathrm{~cm}$ difference between the exposure and the specimens which led to higher $\Delta \mathrm{E}$ value. The same study measured the translucency of the same material and it was $10 \mathrm{TP}$ compared to $7.4 \mathrm{TP}$ in our study [13].

The translucency of teeth differs from tooth to tooth and from area to area at the same tooth, for example, the translucency of the central upper incisor is $15 \mathrm{TP}$ at the incisal area, and it decreases to 5 at the cervical area [19]. The mean TP of $1 \mathrm{~mm}$ thickness human tooth was 16.4 and another study suggests that there are some differences and variations in the TP values of sectioned human dentin with thicknesses ranging from 0.5 to $2.0 \mathrm{~mm}$ [20]. The values of TP in this study reduced significantly under the exposure of the conventional smoking from 10.409 to 7.469 for the nano-hybrid and from 9.922 to 6.907 for the microhybrid composite. Additionally, the electronic cigarettes smoking affected the translucency: it decreased from 9.791 to 6.528 for the micro-hybrid composite and from 10.518 to 7.418 for the nano-hybrid composite. This significant change in the TP values shows that both methods affect the translucency negatively, but the conventional cigarettes have more effect on the translucency than the electronic cigarettes and the micro-hybrid seemed to be the least affected by both methods. The distance between the smoke entrance and the exposed specimens was $8 \mathrm{~cm}$ and it affected the results.

The present study had some limitations because of the in vitro design. In addition, it did not examine multiple brands, concentrations, and flavors of nicotine to further evaluate impact on dental restorative materials or absence of brushing simulation during the exposure of specimens to the smoke. Future studies are recommended on larger sample sizes along with use of different nicotine brands and concentrations. In addition, standardization of number and duration of puffs for all the test groups will be helpful in further validating the results and overcoming some of the limitations of the present study. 


\section{Conclusions}

Within the limitations of this study, we conclude that both conventional cigarettes and electronic cigarettes affect the color and the translucency of composite resins negatively, and they make them appear darker. However, conventional cigarette smoke has more effect on the color stability of the composite resins as compared to electronic cigarettes. From a clinical point of view, the effect of smoke exposure on the tested specimens' color for the time duration to which the specimens were exposed were moderate $(\Delta \mathrm{E}<2)$. The micro-hybrid composites showed better color stability as compared to the nano-hybrid composites.

Author Contributions: Conceptualization, H.A.A. and A.A.E.; methodology, A.A.E., A.A.A.-A., S.S.A. and S.R.H.; software, S.R.H.; validation, H.A.A. and A.A.E.; formal analysis, S.R.H.; investigation, A.A.E., A.A.A.-A., S.S.A. and H.A.A.; resources, H.A.A.; data curation, H.A.A., A.A.E., A.A.A.-A. and S.S.A.; writing-original draft preparation, H.A.A., A.A.E., A.A.A.-A. and S.S.A.; writing-review and editing, A.A.E., A.A.A.-A., S.S.A., S.R.H. and H.A.A.; supervision, H.A.A. and A.A.E.; project administration, A.A.E. All authors have read and agreed to the published version of the manuscript.

Funding: This research received no external funding.

Institutional Review Board Statement: Not applicable.

Informed Consent Statement: Not applicable.

Data Availability Statement: Data are available on request from corresponding author.

Acknowledgments: The authors also are thankful to Nasr Maflehi for helping in the statistical analysis. The research project was approved by the College of Dentistry Research Center (CDRC Reg. \# IR 0296) and funded and supported by CDRC and Deanship of Scientific Research at King Saud University, Riyadh, KSA.

Conflicts of Interest: The authors declare no conflict of interest.

\section{References}

1. Harrell, P.T.; Simmons, V.N.; Piñeiro, B.; Correa, J.B.; Menzie, N.S.; Meltzer, L.R.; Unrod, M.; Brandon, T.H. E-cigarettes and expectancies: Why do some users keep smoking? Addiction 2015, 110, 1833-1843. [CrossRef]

2. Traini, T.; Pettinicchio, M.; Murmura, G.; Varvara, G.; Di Lullo, N.; Sinjari, B.; Caputi, S. Esthetic outcome of an immediately placed maxillary anterior single-tooth implant restored with a custom-made zirconia-ceramic abutment and crown: A staged treatment. Quintessence Int. 2011, 42, 103-108.

3. Pintado-Palomino, K.; de Almeida, C.V.; Oliveira-Santos, C.; Pires-de-Souza, F.P.; Tirapelli, C. The effect of electronic cigarettes on dental enamel color. J. Esthet. Restor. Dent. 2019, 31, 160-165. [CrossRef] [PubMed]

4. Pop-Ciutrila, I.S.; Ghinea, R.; Colosi, H.A.; Dudea, D. Dentin translucency and color evaluation in human incisors, canines, and molars. J. Prosthet. Dent. 2016, 115, 475-481. [CrossRef] [PubMed]

5. Dawkins, L.; Turner, J.; Roberts, A.; Soar, K. 'Vaping'profiles and preferences: An online survey of electronic cigarette users. Addiction 2013, 108, 1115-1125. [CrossRef]

6. ElSayad, I.I. Color and translucency of finished and unfinished esthetic restorative materials after staining and bleaching. Saudi. Dent. J. 2018, 30, 219-225. [CrossRef] [PubMed]

7. Cheng, T. Chemical evaluation of electronic cigarettes. Tob. Control 2014, 23, ii11-ii17. [CrossRef] [PubMed]

8. Schober, W.; Szendrei, K.; Matzen, W.; Osiander-Fuchs, H.; Heitmann, D.; Schettgen, T.; Jörres, R.A.; Fromme, H. Use of electronic cigarettes (e-cigarettes) impairs indoor air quality and increases FeNO levels of e-cigarette consumers. Int. J. Hyg. Environ. Health 2014, 217, 628-637. [CrossRef]

9. Habib, S.R.; Rashoud, A.S.A.; Safhi, T.A.; Almajed, A.H.; Alnafisah, H.A.; Bajunaid, S.O.; Alqahtani, A.S.; Alqahtani, M. Variations in the Shades of Contemporary Dental Ceramics: An In Vitro Analysis. Crystals 2021, 11, 1288. [CrossRef]

10. Mathias, P.; Costa, L.; Saraiva, L.O.; Rossi, T.A.; Cavalcanti, A.N.; da Rocha Nogueira-Filho, G.E. Morphologic texture characterization allied to cigarette smoke increase pigmentation in composite resin restorations. J. Esthet. Restor. Dent. 2010, $22,252-259$. [CrossRef]

11. Goniewicz, M.L.; Knysak, J.; Gawron, M.; Kosmider, L.; Sobczak, A.; Kurek, J.; Prokopowicz, A.; Jablonska-Czapla, M.; RosikDulewska, C.; Havel, C.; et al. Levels of selected carcinogens and toxicants in vapour from electronic cigarettes. Tob. Control 2014, 23, 133-139. [CrossRef]

12. Famele, M.; Ferranti, C.; Abenavoli, C.; Palleschi, L.; Mancinelli, R.; Draisci, R. The chemical components of electronic cigarette cartridges and refill fluids: Review of analytical methods. Nicotine Tob. Res. 2015, 17, 271-279. [CrossRef]

13. Millar, W.J.; Locker, D. Smoking and oral health status. J. Can. Dent. Assoc. 2007, 73, 155. 
14. Ardu, S.; Duc, O.; Di Bella, E.; Krejci, I. Color stability of recent composite resins. Odontology 2017, 105, 29-35. [CrossRef]

15. Hajek, P.; Etter, J.F.; Benowitz, N.; Eissenberg, T.; McRobbie, H. Electronic cigarettes: Review of use, content, safety, effects on smokers and potential for harm and benefit. Addiction 2014, 109, 1801-1810. [CrossRef]

16. Kim, D.; Park, S.H. Color and translucency of resin-based composites: Comparison of A-shade specimens within various product lines. Oper. Den. 2018, 43, 642-655. [CrossRef]

17. Lee, Y.K. Criteria for clinical translucency evaluation of direct esthetic restorative materials. Restor. Dent. Endod. 2016, 41, 159. [CrossRef]

18. IARC Working Group on the Evaluation of Carcinogenic Risks to Humans. Smokeless Tobacco and Some Tobacco-Specific NNitrosamines; IARC Monographs on the Evaluation of Carcinogenic Risks to Humans; WHO: Geneva, Switzerland, 2007; Volume 89, pp. 1-592.

19. Prathap, S.; Rajesh, H.; Boloor, V.A.; Rao, A.S. Extrinsic stains and management: A new insight. J. Acad. Indus. Res. 2013, 1, 435-442.

20. Tan, A.S.; Lee, C.J.; Nagler, R.H.; Bigman, C.A. To vape or not to vape? Effects of exposure to conflicting news headlines on beliefs about harms and benefits of electronic cigarette use: Results from a randomized controlled experiment. Prev. Med. 2017, 105, 97-103. [CrossRef]

21. Vichi, A.; Ferrari, M.; Davidson, C.L. Color and opacity variations in three different resin-based composite products after water aging. Dent. Mater. 2004, 20, 530-534. [CrossRef]

22. Wasilewski, M.D.; Takahashi, M.K.; Kirsten, G.A.; de Souza, E.M. Effect of cigarette smoke and whiskey on the color stability of dental composites. Am. J. Dent. 2010, 23, 4-8.

23. Watts, A.M.; Addy, M. Tooth discoloration and staining: A review of the literature. Br. Dent. J. 2001, 190, 309-316. [CrossRef]

24. Zanetti, F.; Zhao, X.; Pan, J.; Peitsch, M.C.; Hoeng, J.; Ren, Y. Effects of cigarette smoke and tobacco heating aerosol on color stability of dental enamel, dentin, and composite resin restorations. Quintessence Int. 2019, 50, 156-166.

25. Zhuang, Y.L.; Cummins, S.E.; Sun, J.Y.; Zhu, S.H. Long-term e-cigarette use and smoking cessation: A longitudinal study with US population. Tob. Control 2016, 25, i90-i95. [CrossRef]

26. Cadenaro, M.; Marchesi, G.; Antoniolli, F.; Davidson, C.; Dorigo, E.D.; Breschi, L. Flowability of composites is no guarantee for contraction stress reduction. Dental Mater. 2009, 25, 649-654. [CrossRef]

27. Sharafeddin, F.; Motamedi, M.; Fattah, Z. Effect of preheating and precooling on the flexural strength and modulus of elasticity of nanohybrid and silorane-based composite. J. Dent. 2015, 16 (Suppl. S3), 224.

28. Moezizadeh, M.; Mokhtari, N. Fracture resistance of endodontically treated premolars with direct composite restorations. J. Conserv. Dent. JCD 2011, 14, 277. [CrossRef]

29. Tarle, Z.; Attin, T.; Marovic, D.; Andermatt, L.; Ristic, M.; Tauböck, T.T. Influence of irradiation time on subsurface degree of conversion and microhardness of high-viscosity bulk-fill resin composites. Clin. Oral Investig. 2015, 19, 831-840. [CrossRef] [PubMed]

30. Alandia-Roman, C.C.; Cruvinel, D.R.; Sousa, A.B.; Pires-de-Souza, F.C.; Panzeri, H. Effect of cigarette smoke on color stability and surface roughness of dental composites. J. Dent. 2013, 41, e73-e79. [CrossRef] [PubMed]

31. Bazzi, J.Z.; Bindo, M.J.; Rached, R.N.; Mazur, R.F.; Vieira, S.; de Souza, E.M. The effect of at-home bleaching and toothbrushing on removal of coffee and cigarette smoke stains and color stability of enamel. J. Am. Dent. Assoc. 2012, 143, e1-e7. [CrossRef] [PubMed]

32. Barutcigil, C.; Harorli, O.T.; Yildiz, M.; Ozcan, E.; Arslan, H.; Bayindir, F. The color differences of direct esthetic restorative materials after setting and compared with a shade guide. J. Am. Dent. Assoc. 2011, 142, 658-665. [CrossRef] 